\title{
Use of antidepressants in diabetes patients not using drugs, but only monitoring blood glucose
}

\author{
Oivind Hundal ${ }^{1,2 \#}$, Eva Biringer ${ }^{2,3}$, Inge Losnegard ${ }^{4}$, Anders Lund $^{2}$ \\ ${ }^{1}$ Moodnet, Sjukehusapoteka Vest HF, Bergen, Norway; \\ \#Corresponding Author: oivind.hundal@kliniskmedisin.uib.no \\ ${ }^{2}$ Moodnet, Haukeland University Hospital, Bergen, Norway \\ ${ }^{3}$ Psychiatric Clinic, Helse Fonna HF, Stord, Norway \\ ${ }^{4}$ Helse Bergen HF, Bergen, Norway
}

Received 11 January 2012; revised 29 February 2012; accepted 28 March 2012

\section{ABSTRACT}

Objective: Patients with diabetes have an increased Odds Ratio (OR) for depressive disorder. We wanted to investigate if patients with metabolic syndrome and/or diabetes type 2 not treated with antidiabetic agents, have an OR for concurrent antidepressant use comparable to other types of diabetes. Methods: Drug delivery data from 25 pharmacies were analysed with respect to sale of antidepressants, oral antidiabetic agents, insulin and blood glucose test strips. Results: Total population of the area was 337,019 , whereas 254,083 were 18 or older. Of these 20,139 were patients receiving insulin, oral antidiabetics, glucose test strips and/or antidepressants. Those receiving antidepressants were $5.8 \%$ of those 18 or older whereas $2.4 \%$ received any antidiabetic medication (including test strips for HBGM). For patients receiving no medications but test strips alone, the adjusted OR for use concurrent use of antidepressants was $\mathbf{1 . 6 2}$ (95\% confidence interval: $1.19-2.23$ ), $p=0.002$. For insulin-only receiving patients, the adjusted OR was 1.65 (1.41 - 1.93), $p=0.000$, whereas for patients receiving only oral antidiabetics, it was 1.53 (1.38 - 1.71), $p=0.000$. Patients receiving both insulin and oral antidiabetics: 2.23 (1.75 2.85), $p=0.000$. Conclusion: Patients receiving only blood glucose strips have an OR of concurrently receiving antidepressants, not different from those receiving insulin only or oral antidiabetics only.

Keywords: Type 2 Diabetes; Metabolic Syndrome; Blood Glucose Strips; Depression; Comorbidity

"Disclosure: None of the authors have any interests to declare.

\section{INTRODUCTION}

The British physician Thomas Willis in 1684 described diabetes as "sadness or long sorrow and other depressions and disorders". Several studies have addressed the apparent connection between major depressive disorder and diabetes. And this association is now well established for both type 1 and type 2 diabetes [1-4]. There is no clear-cut division between metabolic syndrome and type 2 diabetes. Rather a continuum from metabolic syndrome via diet-only regulated diabetes to diabetes type 2 treated with oral antidiabetics.

We have found no studies that address diabetes type 2 patients that are treated with neither insulin nor oral antidiabetic agents, but only use blood glucose strips for home blood glucose monitoring (HBGM). As these patients do not use drugs as such for their diabetes, they are not found in prescription registries. Manual collection of data on test strips for HBGM is then relevant.

The aims of the present study were: First to describe the Odds Ratio (OR) of use of antidepressants in patients with receiving only insulin, only oral antidiabetics, or receiving blood glucose strips. Second to compare the ORs within the different types of diabetes.

\section{METHODS}

In Norway all drugs for regular and longlasting use are covered by the public health care system. A prerequisite is that the physician uses the authorised "Blue prescription" which is mandatory for drugs reimbursed by the health system. All patients receiving this type of prescription have relevant and confirmed diagnoses. At each delivery the patients receive sufficient medication and/or equipment for 3 months use. Blood glucose strips are covered the same way as insulin and oral antidiabetics. The patients in this study have all been to the pharmacy and obtained their medication and/or blood glucose strips. 
Bergen is the main city in the Western part of Norway. We obtained prescription data from all 25 pharmacies in Bergen and surroundings, for a period of 6 months; February-July 2002. All antidepressant drugs (ATC-group N06A), all oral antidiabetic agents (A10B) and insulins (A10A) were registered. In addition test strips for HBGM were registered. The distance to the nearest pharmacy outside this area, was approximately 10 times the distance to the nearest pharmacy within, making it probable that we selectively covered the population within this area. Only patients at least 18 years of age, were Registered.

All data were delivered on an Excel ${ }^{\circledR}$ spreadsheet. Visual Basic ${ }^{\circledR}$ macros were programmed to edit the raw data, organise the data correctly and remove obvious errors. The macros combined several drug deliveries, eventually from different pharmacies, to each patient, into one case per patient. All further corrections were done by two independent persons and double-checked manually.

To investigate the relationship between purchase of antidiabetic agents (i.e. insulin and/or oral antidiabetics), a logistic regression model with the dichotomous variable for antidiabetic medication (Yes/No) as independent variable and purchase of antidepressant medication (Yes/No) as dependent variable in the first step was applied. Then, the analysis was adjusted for the effects of age and sex in a second step. All tests were two-tiled with an $\alpha$ level of 0.005 . When comparing two ORs, an $\alpha$ level of 0.05 was chosen. Analyses were performed with the statistical pacckage SPSS version 15.00.

The study was approved by the Norwegian Data In spectorate, the Directorate for Health and Social Affairs, and the Regional Committee for Medical Research Ethics, cfr the Helsinki Declaration.

\section{RESULTS}

The population of this area was 168,815 males and 168,204 females. Of these 129,657 females and 124,426 males were 18 years or older. Mean age of males was 44.8 years and standard deviation (SD) was 17.3 years and range 18 -103, whereas mean age of females was 47.4 years (SD 19.2, range 18 - 105). The patients are described in Table 1.

Of the patients with diabetes, $11.6 \%$ received antidepressants whereas of those without, $5.7 \%$ did. Of patients receiving insulin only, oral agents only, both insulin and ora agents and blood glucose strips only, $10.0 \%, 12.0 \%$, $15.6 \%$ and $10.7 \%$, respectively, did receive antidepressants. Of the men with diabetes, $8.2 \%$, and of the women with diabetes, $15.4 \%$ did receive antidepressants.

Odds Ratio (OR) was 2.17 (95\% confidence interval (CI) 2.00; 2.35), $p=0.000$, in the crude analysis of all patients, indicating that use of antidepressants was significantly higher among patients with diabetes than in those who were not. After adjustment for the effects of age and sex, OR for use of antidepressants in diabetics was 1.66 (95\% CI 1.53; 1.80), $p=0.000$. In Table 2 are given adjusted ORs, stratified into types of antidiabetic treatment.

\section{DISCUSSION}

Our main finding is that diabetes type 2 patients receiving only blood glucose strips also are associated with a significant increased antidepressant use, OR comparable to both diabetes type 1 (insulin only) and type 2 (oral antidiabetics only). Whereas insulin-dependent type 2 diabetes had a significantly higher OR. This diabetes not

Table 1. The different patient groups described.

\begin{tabular}{cccccc}
\hline Type patients & $N$ & Mean & SD & Range & Males/females \\
\hline Insulin only & 1797 & 52.5 & 19.7 & $992 / 805$ & 199 \\
Oral antidiabetic agents only & 3228 & 67.6 & 13.8 & $18-97$ & $30-95$ \\
Both insulin and oral antidiabetics & 499 & 64.0 & 12.5 & 18.1 & $18-97$ \\
Blood glucose strips only & 420 & 55.3 & $183 / 237$ \\
\hline
\end{tabular}

Table 2. Odds Ratio (OR) for concurrent use of antidepressants. All ORs were significantly different from 1.00.

\begin{tabular}{ccc}
\hline Drugs received & OR & 95 \% Confidence Interval \\
\hline Insulin only & $1.65^{* \#}$ & $1.41-1.93$ \\
Oral antidiabetic agents only & $1.53^{* \&}$ & $1.38-1.71$ \\
Both insulin and oral antidiabetics & $2.23^{\text {\#\& }}$ & $1.75-2.85$ \\
Blood glucose strips only & $1.62^{\S}$ & $1.19-2.23$ \\
\hline
\end{tabular}

The same symbol for two diabetes types denote significant difference, $p<0.05$. 
treated with medications is not easily discernable from metabolic syndrome. Still in this patient group the OR of antidepressant use is not clinically discernable from that of diabetes patients using oral agents only. To some degree, this supports the view that depression has a association to disturbances in glucose metabolism [5].

There must be a verified diagnosis of type 2 diabetes in order to have these blood glucose strips reimbursed, cfr the requirements for the "blue prescription". We may assume that those receiving blood glucose strips only and no antidiabetics at all are treated by diet and/or exercise alone. Although there is a continuum from metabolic syndrome to frank type 2 diabetes, the lack of use of oral antidiabetic agents discerns these patients from other forms of type 2 diabetes. Golden et al. [6] found that patients with type 2 diabetes treated with oral antidiabetics, actually had higher risk of becoming depressed compared to patients with impaired fasting serum glucose, and patients with untreated type 2 diabetes. Kohen and Lester [7] reported that the antidiabetic exenatide induced depression in a 70 year old woman successfully treated with fluoxetine. This possible pharmacologically induced depression effect is not present in the diet/exercise-only diabetes group. It must, however, be taken into account that the registrations only covered 6 months. And the patients might have been using oral antidiabetics earlier. As the patients covered by the governmental reimbursement only receive drugs and/or test strips for 3 months, we may assume that these patients do not use insulin nor any oral antidiabetics. At least not in the registration period.

There are published numerous studies on the connection between metabolic syndrome and depression. Several studies show the association between insulin resistance and depression [8-10]. Heiskanen et al. 2006 [11] found significant higher prevalence of metabolic syndrome in patients earlier treated for depression. Those who still were treated for depression had an even higher prevalence. Spuriously, Vogelzangs et al. 2011 [12] found that abdominal obesity; waist circumference, but not metabolic syndrome, predicted the onset of depression. Length of depression and number of episodes do not influence the risk for metabolic resistance [13].

Diabetes patients receiving both insulin and oral antidiabetics most probably have a more severe type 2 diabetes; the patients have not achieved their therapeutic goal with oral antidiabetics alone, and adjuvant insulin was necessary. Most probably these patients with combination therapy suffer from diabetes type 2, not type 1 . Oral antidiabetics are reimbursed for use in diabetes type 2 only. This might be interpreted that the possible increased severity of this diabetes constitutes the increased OR for antidepressant use. Several studies support the idea that prolonged hyperglycemia/poorly regulation is associated with depression [14-16].

The main strength of this study is that all patients, both patients with diabetes and patients suffering from depressive disorder, have a verified diagnosis and are treated with drugs on a regular and longlasting basis. Cfr the requirements for the "blue prescription". In addition, this study is unique in that it presents data on and compares four diabetes treatments. As the data are unselected; all deliveries of antidepressants, antidiabetics and/or blood glucose strips were registered, the chance for a selection bias is low.

We can not make the assumption that all antidepressants were used as treatment for depresive disorder. Hence, it is a limitation of the study is that antidepressants also are used for bipolar disorder and anxiety. The co-morbidity between depression and anxiety is substantial. A systematic review of 18 studies found a prevalence of anxiety between $1.3 \%$ and $26.5 \%$ depending on specific diagnosis of depression [17]. Das-Munshi et al. [18] found in 8580 individuals with diabetes an OR of 1.9 for depression, 1.3 for anxiety and 1.7 for comorbid anxiety and depression. There also is a possibility that patients regularly visiting their doctor for control of their diabetes, might have a greater probability that their depression is recognised and treated, cfr the Berkson paradox [19]. Our data origin in deliveries of drugs from a pharmacy upon presentation of a physician's prescription. Thus, the drugs have been delivered to the patients. Hence, OR data do not encompass drugs given in hospitals and nursing homes. It is, however, very seldom that people are hospitalized for several months. The missing patients from nursing homes will affect the oldest group only. The study only shows the point prevalence of the connection between diabetes treatment and antidepressant treatment.

We have shown that all main types of diabetes are associated with an increased OR for use of antidepressant drugs. And that diabetes type 2 treated with neither insulin nor oral antidiabetic agents, have a comparable increased OR for receiving antidepressants. And our study extends and supports earlier studies on metabolic syndrome/diabetes type 2 and depression.

\section{ACKNOWLEDGEMENTS}

The authors are highly thankful to Mrs Anna S. Hundal for extensive help and cooperation in controlling and editing the raw data. We also thank the 25 proprietary pharmacists for extensive help in collecting the data.

\section{REFERENCES}

[1] Gavard, J., Lustman, P. and Clouse, R. (1993) Prevalence of depression in adults with diabetes: An epidemiological evaluation. Diabetes Care, 16, 1167-1178. 
doi:10.2337/diacare.16.8.1167

[2] Anderson, R., Freedland, K., Clouse, R. and Lustman, P. (2001) The prevalence of co-morbid depression in adults with diabetes. Diabetes Care, 24, 1069-1078. doi:10.2337/diacare.24.6.1069

[3] Barnard, K., Skinner, T. and Peveler, R. (2006) The prevalence of co-morbid depression in adults with type 1 diabetes: Systematic literature review. Diabetic Medicine, 23, 445-448. doi:10.1111/j.1464-5491.2006.01814.x

[4] Ali, S., Stone, M., Peters, J., Davies, M. and Khunti, K. (2006) The prevalence of co-morbid depression in adults with type 2 diabetes: A systematic review and metaanalysis. Diabetic Medicine, 23, 1165-1173. doi:10.1111/j.1464-5491.2006.01943.x

[5] Hundal, O. (2007) Major depressive disorder viewed as a dysfunction in astroglial bioenergetics. Medical Hypotheses, 68, 370-377. doi:10.1016/j.mehy.2006.06.050

[6] Golden, S., Lazo, M., Carnethon, M., Bertoni, A., Schreiner, P., Roux, A., Lee, H. and Lyketsos, C. (2008) Examining a bidirectional association between depressive symptoms and diabetes. Journal of the American Medical Association, 299, 2751-2759. doi:10.1001/jama.299.23.2751

[7] Kohen, I. and Lester, P. (2008) Exenatide-induced depression in a geriatric patient. International Journal of Geriatric Psychiatry, 23, 443-444. doi:10.1002/gps.1937

[8] Lawlor, D., Smith, G. and Ebrahim, S. (2003) Association of insulin resistance with depression: Cross sectional findings from the British women's heart and health study. British Medical Journal, 327, 1383-1384. doi:10.1136/bmj.327.7428.1383

[9] Timonen, M., Laakso, M., Jokelainen, J., Rajala, U., MeyerRochow, V. and Keinänen-Kiukaanniemi, S. (2005) Insulin resistance and depression: Cross sectional study. British Medical Journal, 330, 17-18. doi:10.1136/bmj.38313.513310.F71

[10] Everson-Rose, S., Meyer, P., Powell, L., Pandey, D., Torréns, J., Kravitz, H., Bromberger, J. and Matthews, K. (2004) Depressive symptoms, insulin resistance, and risk of diabetes in women at midlife. Diabetes Care, 27, 2856-2862. doi:10.2337/diacare.27.12.2856

[11] Heiskanen, T., Niskanen, L., Hintikka, J., KoivumaaHonkanen, H., Honkalampi, K., Haatainen, K. and Vii- namäki, H. (2006) Metabolic syndrome and depression: A cross-sectional analysis. Journal of Clinical Psychiatry, 67, 1422-1427. doi:10.4088/JCP.v67n0913

[12] Vogelzangs, N., Beekman, A., Boelhouwer, I., Bandinelli, S., Milaneschi, Y., Ferrucci, L. and Penninx, B. (2011) Metabolic depression: A chronic depressive subtype? Findings from the InCHIANTI study of older persons. Journal of Clinical Psychiatry, 72, 598-604. doi:10.4088/JCP.10m06559

[13] Goldbacher, E., Bromberger, J. and Matthews, K. (2009) Lifetime history of major depression predicts the development of the metabolic syndrome in middle-aged women. Psychosomatic Medicine, 71, 266-272. doi:10.1097/PSY.0b013e318197a4d5

[14] Lustman, P., Clouse, R., Ciechanowski, P., Hirsch, I. and Freedland, K. (2005) Depression-related hyperglycemia in type 1 diabetes: A mediational approach. Psychosomatic Medicine, 67, 195-199. doi:10.1097/01.psy.0000155670.88919.ad

[15] Skaff, M., Mullan, J., Hoffman, L., Mohr, D., Almeida, D., Masharani, U. and Fisher, L. (2009) Daily negative mood affects fasting glucose in type 2 diabetes. Health Psychology, 28, 265-272. doi:10.1037/a0014429

[16] Richardson, L., Egede, L., Mueller, M., Echols, C. and Gebregziabher, M. (2008) Longitudinal effects of depression on glycemic control in veterans with type 2 diabetes. General Hospital Psychiatry, 30, 509-514. doi:10.1016/j.genhosppsych.2008.07.001

[17] Grigsby, A., Anderson, R., Freedland, K., Clouse, R. and Lustman, P. (2002) Prevalence of anxiety in adults with diabetes: A systematic review. Journal of Psychosomatic Research, 53, 1053-1060. doi:10.1016/S0022-3999(02)00417-8

[18] Das-Munshi, J., Stewart, R., Ismail, K., Bebbington, P., Jenkins, R. and Prince, M. (2007) Diabetes, common mental disorders, and disability: Findings from the UK National Psychiatric Comorbidity Survey. Psychosomatic Medicine, 69, 543-550. doi:10.1097/PSY.0b013e3180cc3062

[19] Berkson, J. (1946) Limitations of the application of fourfold table analysis to hospital data. Biometric Bulletin, 2, 247-253. doi: $10.2307 / 3002000$ 